\title{
The influence of artificial paths and landmarks on the foraging behavior of Norway rats (Rattus norvegicus)
}

\author{
JOHN P. ROCHE and WILLIAM TIMBERLAKE \\ Indiana University, Bloomington, Indiana
}

\begin{abstract}
In two experiments, we explored how the foraging behavior of Norway rats was influenced by different arrangements of artificial paths and vertical landmarks. The rats used the paths successively less for orienting to food in treatments in which paths led to food but were crooked, in which paths led only halfway to food, and in which paths were misaligned with respect to food. The arrangement of paths influenced the rats' rate of energy intake in the beginning of the experiment, whereas the arrangement of beacons did not. With experience, the rats employed different orientation strategies in the presence of different arrangements of paths or beacons, and, by the final 4 days, all groups achieved statistically indistinguishable net rates of return. The rates of energy intake were similar because the rats in different treatments traveled similar distances per session, despite differing arrangements of paths and landmarks.
\end{abstract}

Colonies of Norway rats (Rattus norvegicus) in large outdoor enclosures tend to follow trails above the ground (Calhoun, 1962), and rats are seldom seen off of these trails (Telle, 1966). The above-ground trails, or paths, are often drawn toward vertical landmarks, such as trees and fenceposts, and they frequently lead to food sites (Calhoun, 1962). One factor that could have contributed to the evolution of following paths and approaching vertical landmarks is that to do so may allow rats to find food efficiently. Other factors that could have favored path following and landmark approach are involved with predator avoidance; these behaviors may offer direct protection from predators (see Calhoun, 1962) or provide known routes for escape from predators (see, e.g., Fanselow \& Lester, 1988).

Conceptual support for the possibility that path following contributes to foraging efficiency is provided by the observation that because energy (food) often occurs in discrete clumps or "patches" within the environment (MacArthur \& Pianka, 1966; Roche, 1996; Roche, Stubbs, \& Glanz, 1996; Stephens, 1990; Stephens \& Krebs, 1986), achieving a high net rate of energy intake frequently relies, in part, on gathering information about the location of food

Thanks to Tammi Nelson and Jason Hesting for their technical assistance. Special thanks to Cynthia Hoffman, for helping to pioneer this approach and for making illustrations of the apparatus, and to Joseph Leffel, for all of his invaluable contributions. David Sherry, Francisco Silva, Joseph Leffel, Susan Thomas, Gary Lucas, and two anonymous reviewers offered helpful comments on earlier versions of this manuscript. This study was supported in part by National Science Foundation Grants IBN 9408366 and BIR-9413220. Correspondence concerning this article should be addressed to $J$. P. Roche, Center for the Integrative Study of Animal Behavior, 402 North Park Ave., Indiana University, Bloomington, IN 47405 (e-mail: jroche@indiana.edu).

-Accepted by previous editor, Robert A. Rescorla sites and navigating among those food sites effectively (Bell, 1991). Thus, traits for path following or for landmark use may have been selected because these traits allowed rats to orient in the environment and to economically gather food from spatially separated food patches.

Empirical support for the hypothesis that rats can use paths to economically forage for food has been provided by laboratory research that uses radial arm mazes in which rats forage from a central platform that is surrounded by a radial array of artificial paths (arms) that lead to food sites. Rats forage economically, in terms of making few repeat visits to previously visited arms, in these studies when food is placed at the end of each arm (see, e.g., Ash \& Roberts, 1992; Olton \& Samuelson, 1976; Schacter, Phelps, Brodbeck, Mogenson, \& Roberts, 1991). Radial arm mazes generally constrain rats to remain on maze arms, but rats have been observed to continue to show a pronounced predisposition to remain on paths even when they can readily get off of them. For example, Hoffman, Timberlake, Gont, and Leffel (1997) placed naive rats in an open arena in which there was a radially arranged hexagon of artificial paths on the floor that led from a central location to food. Hoffman et al. observed that, although the rats frequently got off of the paths, they remained on the paths for more than $70 \%$ of the total distance that they traveled.

The purpose of the present study was to explore how path following and landmark orientation contributed to foraging efficiency when Norway rats were presented with different arrangements of artificial paths and vertical landmarks (beacons) in an arena in which rats were free to get on and off paths. In Experiment 1, we investigated the influence that the directness with which artificial paths lead to food has on foraging efficiency and the extent to which these paths are used as orientation cues. In Experiment 2, we investigated the influence that the directness with 
which beacons lead to food has on foraging efficiency, the relative influence of paths versus beacons on foraging efficiency, and the extent to which rats tend to approach beacons when those beacons do not lead to food.

\section{EXPERIMENT 1 Paths}

In Experiment 1, we explored the relationship between path following and foraging efficiency with different arrangements of artificial paths in a $3.66-\mathrm{m}^{2}$ arena in which there were six food cups equidistant from a central location. We investigated the influence that the directness with which paths lead to food has on foraging efficiency by comparing the rate of energy intake in an environment in which paths led directly to food with that in an environment in which paths were misaligned with respect to food. The extent to which different arrangements of paths are used as orientation cues for approaching food was in- vestigated by comparing the number of approaches to food that were made from paths in environments in which paths led to food, in which paths were crooked (and thus more costly to follow), in which paths were misaligned with respect to food, and in which paths were short and thus reached only halfway to food.

\section{Method}

Subjects. Thırty-four adult, female, Sprague-Dawley Norway rats served as subjects. All of the rats were experimentally naive at the beginning of the study, and each experimental treatment used a different group of rats. The animals were housed singly in stainless steel cages in a colony with a 12:12-h light:dark schedule. Rats were run during the light phase of this cycle. Water was always available in the home cages. The rats were maintained at $85 \%$ of their freefeeding werghts; in order to maintain this weight level, additional food (Purina Rat Chow) was provided in their home cages $30 \mathrm{~min}$ after the last rat in an experimental treatment was run.

Apparatus. Experimental trials were conducted in a $3.66-\mathrm{m}^{2}$ arena with a gray cement floor. Three sides of the arena were bounded by $91.4-\mathrm{cm}$-high wooden barriers (painted grey), and the

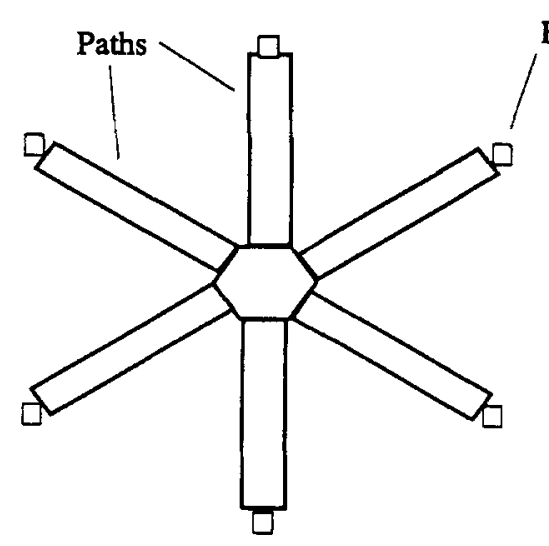

PATHS-TO-FOOD

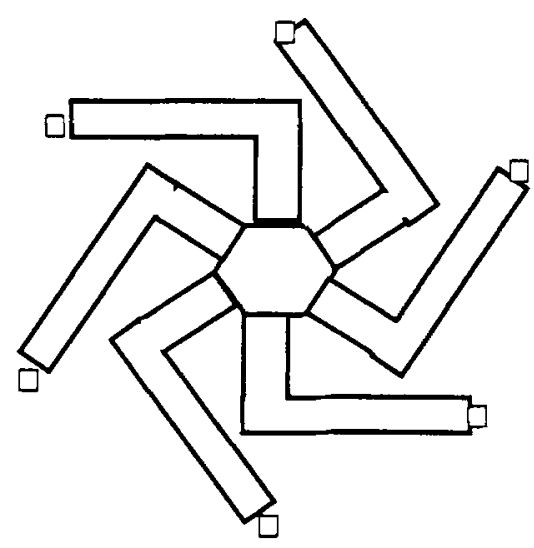

CROOKED PATHS

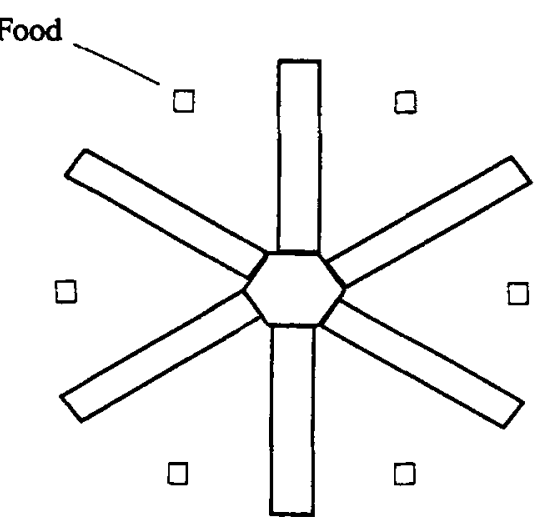

MISALIGNED PATHS

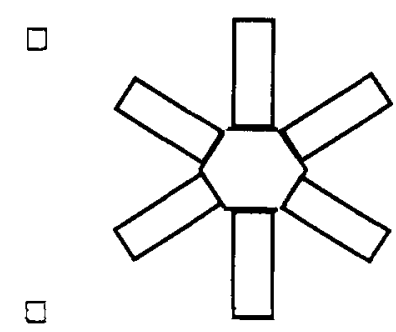

SHORT PATHS

Figure 1. The configuration of paths in experimental treatments in Experiment 1. 
fourth side was bounded by a wall (all four sides of the arena will be henceforth referred to as walls). The room was dimly lit with a bare $60-\mathrm{W}$ bulb that shone from the ceiling above the center of the maze.

In each experimental treatment, six food cups, $7.5 \mathrm{~cm}$ in diameter and $5 \mathrm{~cm}$ in depth, were arranged in a hexagonal pattern in which each cup was $91.4 \mathrm{~cm}$ from adjacent cups. Paths (arms), which lay directly on the floor of the arena, were wooden, and they were painted gray. Paths were $10.2 \mathrm{~cm}$ wide, had $3.8-\mathrm{cm}$-high sidewalls, and had floors $1.9 \mathrm{~cm}$ in thickness.

In every treatment, there was a center structure in the middle of the arena. The center structure, which was a wooden hexagon painted gray, measured $45 \mathrm{~cm}$ in diameter. A clear hexagon of Plexiglas was mounted $15 \mathrm{~cm}$ above the center structure. The Plexiglas was half transparent and half opaque. There was a series of $2.5-\mathrm{cm}$-wide black construction paper strips spaced $2.5 \mathrm{~cm}$ apart across the top of the Plexiglas. These strips were oriented in the same way each day. In treatments in which paths were present, the paths were attached to the center along the flat portions of the center hexagon. The areas of the center hexagon that did not connect to paths had 3.8-cm sidewalls. These sidewalls were also in place when there were no paths. The wood floor of the center structure was $1.9 \mathrm{~cm}$ in thickness.

Procedure. Experimental trials were conducted between 1100 and 1700 EST 6 days per week. Each subject was run in 1 trial per day for a total of 14 trials, and individual experimental treatments were run in the same 2-h time period each day. The experimenter carried four rats at a time to the experimental room in a rat carrier that contained four $10 \times 20 \mathrm{~cm}$ compartments. The order in which the rats were run in individual experimental treatments was systematically rotated on different days. Prior to each session, the experimenter placed one 45-mg Noyes pellet in each cup. At the beginning of each experimental trial, the experimenter placed an individual rat in the center structure (orienting the rat away from the experimenter) and then the experimenter moved out of the arena to an observation seat near one corner of the arena. The pattern of movement of the rat was recorded on a sheet of paper that had a grid and an outline of the particular arrangement of food cups, paths, and beacons. The experimenter also recorded the amount of time that the rat took to make eight cup visits. Each session ended after $5 \mathrm{~min}$ had passed, after eight total cup visits had been made, or after six unique cups had been visited and 1 additional minute had passed, whichever came first. The expermenter then removed the rat from the arena and began another trial with a different rat. After every fourth subject, the entire arena was mopped with detergent solution and dried to remove odor residues. Individuals that failed to move from the center structure by the sixth day were removed from the study (each treatment group began with 10 rats).

The four experimental treatments in Experiment 1 were as follows (Figure 1): (1) paths to food-six 91.4-cm-long paths led from the center of the maze directly to the six food cups $(n=8)$; (2) misaligned paths-six 91.4-cm-long paths led from the center of the maze to the region equidistant between food cups $(n=7)$; (3) short paths-six 45.7-cm paths led from the center directly toward food cups, but stopped $45.7 \mathrm{~cm}$ from the cups $(n=9)$; and (4) crooked paths six paths led from the center $45.7 \mathrm{~cm}$ toward a food cup, then turned at a $90^{\circ}$ angle and led to the left directly to the adjacent food cup, for a total length of $137.2 \mathrm{~cm}(n=10)$. The length of the paths in the paths-to-food treatment was $50 \%$ longer than in the short-paths treatment and $33 \%$ shorter than in the crooked-paths treatment.

We assessed session-wide foraging efficiency by calculating the traditional radial-arm maze measure of the number of novel cups visited in the first six cup visits (number of pellets per six cup visits), and we also calculated the number of pellets eaten per meter traveled (pellets $/ \mathrm{m}$ ). Potential currencies for assessing foraging effectiveness include the following: (1) the gross energy intake, or yield; (2) the energy intake minus the expenditure; (3) the energy intake per unit time or per unit distance (the rate of energy intake); or
(4) the energy intake minus expenditure, divided by the time elapsed or the distance traveled (the net rate of er rgy intake). The currency that is likely to be most closely linked to reproductive success is the net rate of energy intake (see Schoener, 1971). We did not measure the rats' energy expenditure in the arena, but, given the assumption that energy expenditure would be relatively unvarying as the rats traveled, the pellets per meter should correlate closely with the net rate of energy intake. The rats always ate the pellets that were in the cups that they visited, they generally traveled throughout most or all of each session, and their distance traveled was consistently highly correlated with time elapsed.

Localized efficiency of movement in relation to food cups was assessed by calculating the number of instances in which the rats moved directly from one food cup to an adjacent food cup (direct crosses). To obtain a measure of cup-to-cup crosses that were less direct, we measured the number of instances in which the rats traveled across the open from one food cup to an adjacent cup in a route that was indirect (not a straight line between cups) and did not travel to within $45.7 \mathrm{~cm}$ of the edge of the center hexagon or to within $22.9 \mathrm{~cm}$ of a wall (indirect crosses).

Orientation to cups from paths was assessed by calculating the following: (1) the number of instances in the paths-to-food and crooked-paths treatments in which a rat approached a food cup from a path and (2) the number of instances in which the rats in the shortpaths and misaligned-paths treatments moved from the center of the arena, down an entire path, and then moved across the open directly to the closest food cup.

Measurement of distance traveled provided an accurate measure of the proportion of travel on or next to paths, along walls, or in the open. The rats were considered to be following a path if they moved on the path or within $10.2 \mathrm{~cm}$ of the path for at least $15.2 \mathrm{~cm}$. The rats were considered to be following a wall if they traveled parallel to a wall within $22.9 \mathrm{~cm}$ of the wall for more than $45.7 \mathrm{~cm}$. Travel in the open was all travel that was not path following or wall following. The length of each path recorded on the paper grid was measured twice with a pen mouse and Mouse Odometer software on an IBM personal computer. The mean of these two measurements was adjusted to scale.

All variables were calculated up to and including the sixth cup visit. Data from individual days for individual rats were combined into means for 2-day blocks. Comparisons of means among groups of treatments were performed with a one-way analysis of variance (ANOVA) (Sokal \& Rohlf, 1995). Pairwise comparisons were performed with Tukey tests. The relations between the number of novel cups in the first six cup visits and the number of pellets per meter and between the number of direct crosses and the number of pellets per meter were determined with linear regression. Unless otherwise noted, statistical tests were for behavior in the final two 2-day blocks of the experiment; the rats seemed to have reached relatively steadystate behavior by that time. We arcsine transformed proportions before conducting statistical tests on them. Differences were considered significant at an alpha level of .05 .

\section{Results}

General behavior. The rats readily explored the experimental arena, traveling on the paths, in the open, and along the walls. In the paths-to-food treatment, a mean of $66 \%$ of the distance the rats traveled was on the paths in the last 4 days of the experiment. Thus, they allocated a large proportion of their distance traveled to paths, but they also traveled off the paths. In all treatments, the rats increased their number of pellets per six cup visits (Figure 2) and their number of pellets per meter (Figure 3), which indicates that the rats increased their foraging efficiency with experience. These two measures displayed 


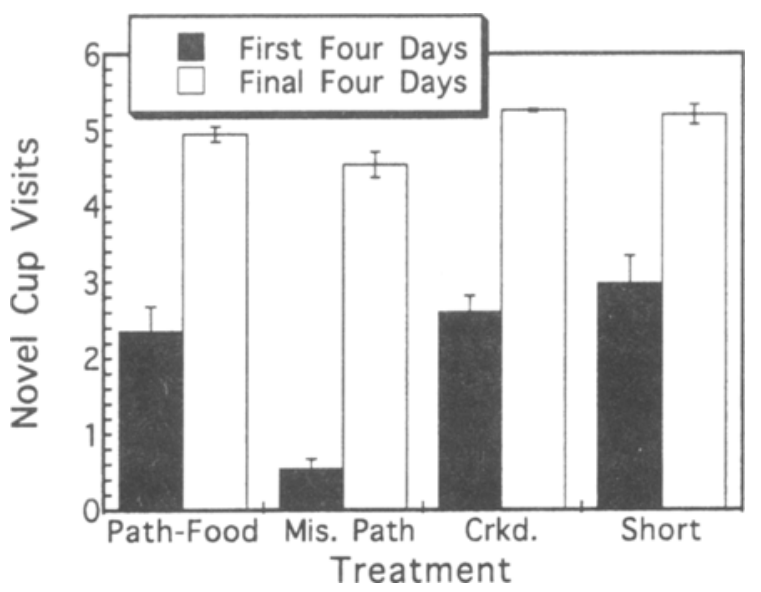

Figure 2. The mean number of novel cups visited up to the sixth cup visit in the first 4 and final 4 days of Experiment 1 (PathFood, paths to food; Mis. Path, misaligned paths; Crkd., crooked paths; Short, short paths).

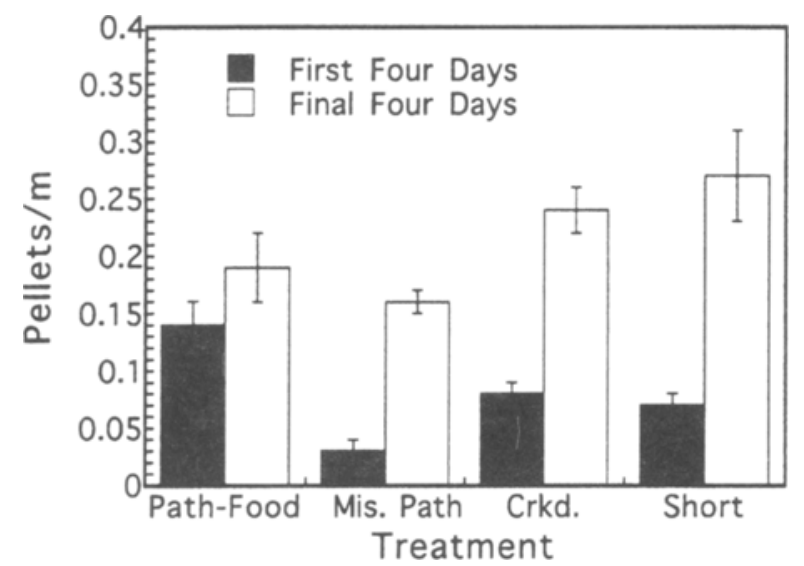

Figure 3. The mean number of pellets per meter in the first 4 and final 4 days of Experiment 1 (Path-Food, paths to food; Mis. Path, misaligned paths; Crkd., crooked paths; Short, short paths).

significant correlations in 33 of 34 rats, a result of the similar distances traveled in all treatments. The mean total distance traveled in the last 4 days did not differ among treatments $[F(3,30)=0.608, p=.615]$. For conciseness, we discuss below only the number of pellets per meter.

The influence of paths on foraging efficiency. There was a significant difference in the mean pellets per meter among treatments in the first 4 days of the experiment $[F(3,30)=7.731, p=.001]$. The rats in the paths-to-food treatment achieved a significantly higher initial number of pellets per meter than that achieved by the rats in the misaligned-paths treatment (Tukey pairwise comparison, $p<.001)$ and by the rats in the short-paths treatment $(p<$ .028 ), but not than that achieved by the rats in the crookedpaths treatment $(p<.083$; Figure 3$)$. By the last 4 days of the study, however, there were no significant differences in the mean number of pellets per meter among treatments $[F(3,30)=2.109, p=.120]$.

The mean number of direct crosses differed among the treatments $[F(3,30)=10.148, p<.001$; Figure 4]. The rats in the short-paths treatment displayed significantly more direct crosses in the last 4 days than did the rats in the paths-to-food $(p<.001)$, misaligned-paths $(p=.007)$, and crooked-paths $(p=.001)$ treatments. The rats in the short-paths treatment may have followed a cross-based strategy, whereas the rats in the paths-to-food treatment employed a path-based strategy, in order to achieve similar rates of energy intake.

The use of paths as orientation cues. Figure 5 displays the mean number of instances, across 2-day blocks, in which the rats used paths to approach food cups (i.e., when the rats approached cups on paths in the paths-tofood and crooked-paths treatments, when the rats followed the entire length of a path and then traveled directly to an adjacent food cup in the misaligned-paths treatment, and when the rats traveled down an entire path and then went straight to the cup to which the path pointed in the shortpaths treatment). In the final 4 days, the rats in different treatments approached cups from paths to differing extents $[F(3,28)=65.513, p<.001]$. The mean number of approaches along paths was higher in the paths-to-food treatment than in the crooked-paths treatment $(p<.001)$, indicating that, when the paths were crooked, the rats used them to approach food cups less than they did when the paths were not crooked. In the final 4 days, the mean number of approaches from paths was significantly higher in the short-paths treatment than in the misaligned-paths treatment $(p=.026)$. The number of approaches from paths was numerically higher in the crooked-paths treatment than in the short-paths treatment, but this difference was not significant $(p=.758)$.

Figure 6 shows that there were differences in the mean proportion of path following among the treatments with

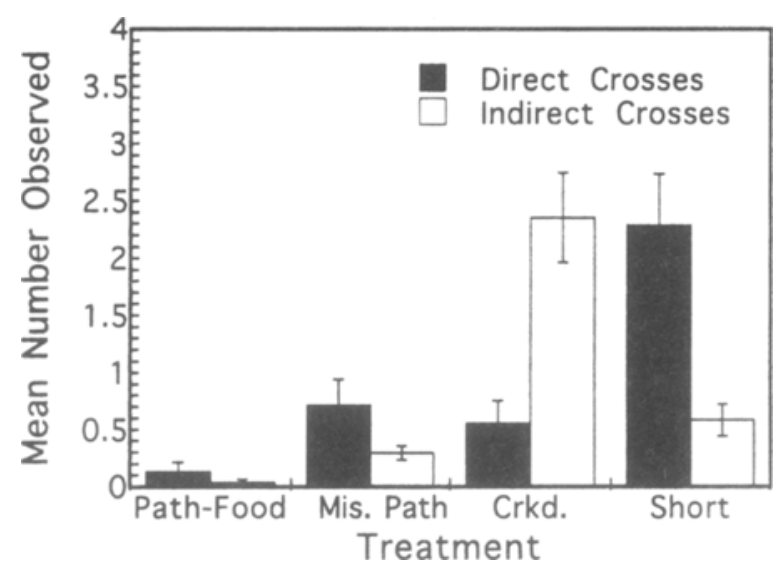

Figure 4. The mean number of direct and indirect crosses observed up to the sixth cup visit in the final 4 days of Experiment 1 (Path-Food, paths to food; Mis. Path, misaligned paths; Crkd., crooked paths; Short, short paths). 


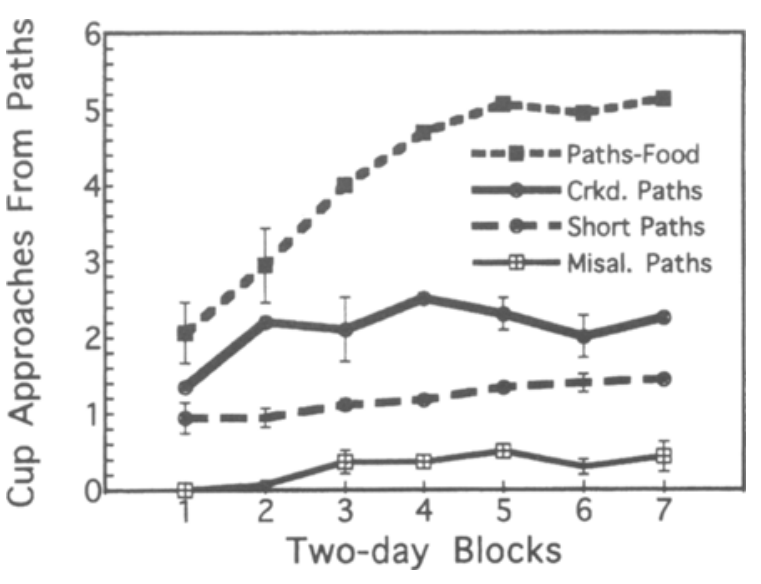

Figure 5. The mean number of cup approaches from paths across 2-day blocks in treatments in Experiment 1 (Paths-Food, paths to food; Crkd. Paths, crooked paths; Short Paths, short paths; Misal. Paths, misaligned paths).

paths in the final 4 days $[F(3,30)=10.989, p<.001]$. The mean proportion of path following in the paths-to-food treatment was significantly higher than in the misalignedpaths treatment $(p<.009)$.

The mean proportion of travel in the open differed among treatments $[F(3,30)=22.324, p<.001]$, even though the total length of paths was $50 \%$ longer in the crooked-paths treatment than in the paths-to-food treatment, and the amount of open space between paths was therefore less in the crooked-paths treatment than in the paths-to-food treatment (Figure 6). There was no significant difference in the proportion of path following between the paths-tofood treatment and the crooked-paths treatment in the final 4 days. However, the proportion of travel in the open was significantly higher in the crooked-paths treatment than in the paths-to-food treatment $(p=.021)$. The rats in the crooked-paths treatment traveled from the center hexagon down the first segment of a path and then straight out

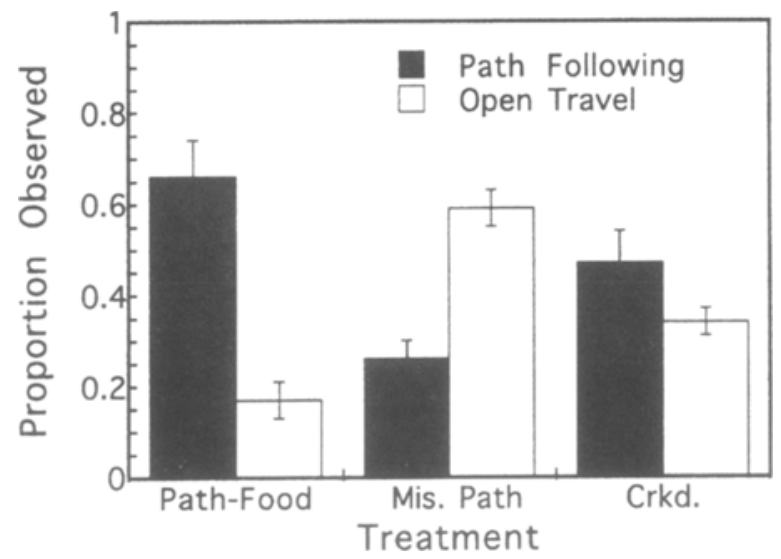

Figure 6. The mean proportion of path following and open travel in the final 4 days of Experiment 1 (Path-Food, paths to food; Mis. Path, misaligned paths; Crkd., crooked paths). to a food cup across the open (i.e., along the route of paths in the paths-to-food treatment) a mean of only $1.1( \pm 0.2)$ times per session.

The number of indirect crosses differed among treatments $[F(3,30)=18.126, p<.001]$. The mean number of indirect crosses was significantly higher in the crookedpaths treatment than in the paths-to-food treatment $(p<$ .001 ), which is further evidence that, when the paths were crooked, the rats traveled between food cups off the paths more frequently than they did when the paths were not elongated (Figure 4).

Linear regressions between the mean number of direct crosses and the mean number of pellets per meter in the final 4 days in the individual treatments revealed the following: These variables showed significant positive correlations in treatments in which there were no paths and in treatments in which paths did not intersect the area between food cups, but they showed no positive correlations in the other treatments (Table 1).

\section{Discussion}

The influence of paths on foraging efficiency. In the first 4 days of the experiment, the rats in the misalignedpaths treatment displayed a lower number of pellets per meter than did the rats in the paths-to-food treatment, which suggests that how directly paths approach food sites influences initial foraging effectiveness. This difference in the number of pellets per meter disappeared by the final 4 days of the experiment, however; this indicates that, with experience, the rats learned to overcome the effect of paths that did not lead to food.

The use of paths as orientation cues. Straight paths that led to food, crooked paths that led to food, misaligned paths, and short paths had successively less importance as orientation cues for finding food.

Similarly, when the paths were misaligned, the rats traveled less on the paths than they did when the paths led to food. When the paths were crooked, the rats traveled more across the open between paths and approached cups less frequently from paths than did the rats in the paths-tofood treatment. These observations indicate that the degree of path following is sensitive to how directly those paths lead to food. If path following were based entirely on predator avoidance, the rats in the misaligned-paths treatment would have been expected to remain on the paths as much as did the rats in the paths-to-food treatment, and the rats in the crooked-paths treatment would not be expected to travel more in the open than did the rats in the paths-tofood treatment.

\section{EXPERIMENT 2 Beacons}

In Experiment 2, we explored the influence that local vertical landmarks (beacons) have on foraging effectiveness by comparing the foraging efficiency of rats in an environment in which beacons led directly to food with that in an environment in which beacons were misaligned with 
Table 1

Results of Linear Regressions of the Mean Number of Direct Crosses in Individual Rats and the Mean Number of Pellets per Meter Observed in Those Individual Rats in the Final 4 Days of Experiments 1 and 2

\begin{tabular}{lcrr}
\hline \multicolumn{1}{c}{ Treatment } & $r$ & $n$ & $p$ \\
\hline Paths to food & .373 & 8 & .362 \\
Misaligned paths & .045 & 7 & .924 \\
Crooked paths & .147 & 10 & .686 \\
Short paths & .939 & 9 & $<.001$ \\
Paths and beacons & .280 & 9 & .465 \\
Beacons to food & .939 & 10 & $<.001$ \\
Misaligned beacons & .872 & 10 & .001 \\
\hline
\end{tabular}

respect to food. We examined whether the rats displayed a predisposition to approach beacons by observing how often rats in an environment in which beacons did not lead to food approached to within $15 \mathrm{~cm}$ of a beacon. We investigated the influence that paths have on foraging efficiency and the relative influence that paths, beacons, and paths plus beacons have on foraging efficiency by making the following comparisons: (1) the rate of energy intake of rats in an environment in which both paths and beacons led to food with that of rats in an environment in which only beacons led to food; (2) the rate of energy intake of rats in an environment in which beacons led to food with that of rats in an environment in which only paths led to food (the paths-to-food treatment from Experiment 1); and (3) the rate of energy intake of rats in an environment in which paths led to food with that of rats in an environment in which both paths and beacons led to food.

\section{Method}

Subjects. Twenty-nine adult, female, Sprague-Dawley Norway rats served as subjects. The subjects were housed as described in Experiment 1 .

Apparatus. The apparatus was the same as that described in Experiment 1 , with the addition of beacons. Beacons were $45.7-\mathrm{cm}$-tall lengths of $2.5-\mathrm{cm}$-diameter wooden doweling attached to a $15.2 \times$ $15.2 \times 1.9 \mathrm{~cm}$ piece of wood. The base of each beacon was painted gray, and the tower of each beacon was covered with black and white stripes $1.9 \mathrm{~cm}$ in width.

Procedure. The procedure, data collection, and statistical analysis were the same as those in Experiment 1. We conducted the following treatments with beacons (Figure 7): (1) beacons to food - six beacons were directly next to the food cups and no paths were present $(n=10)$; (2) misaligned beacons - six beacons were equidistant from nearby food cups and $45.72 \mathrm{~cm}$ outward from the cross be-

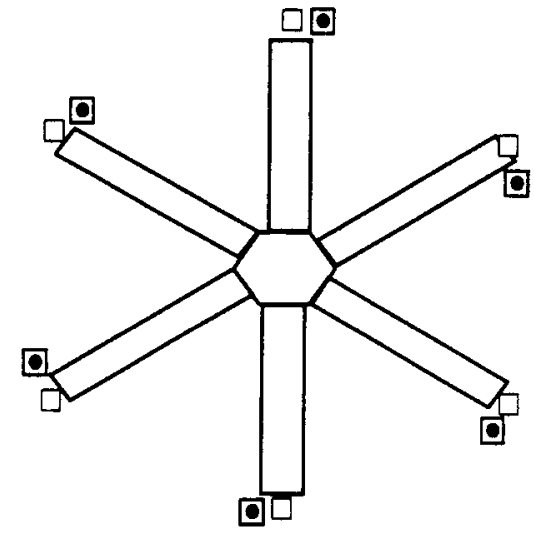

PATHS+BEACONS

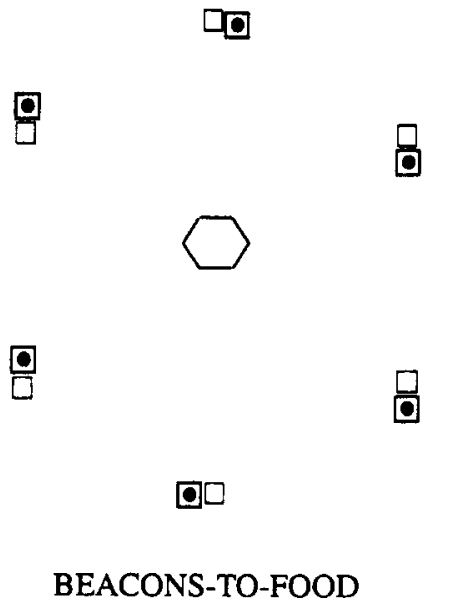

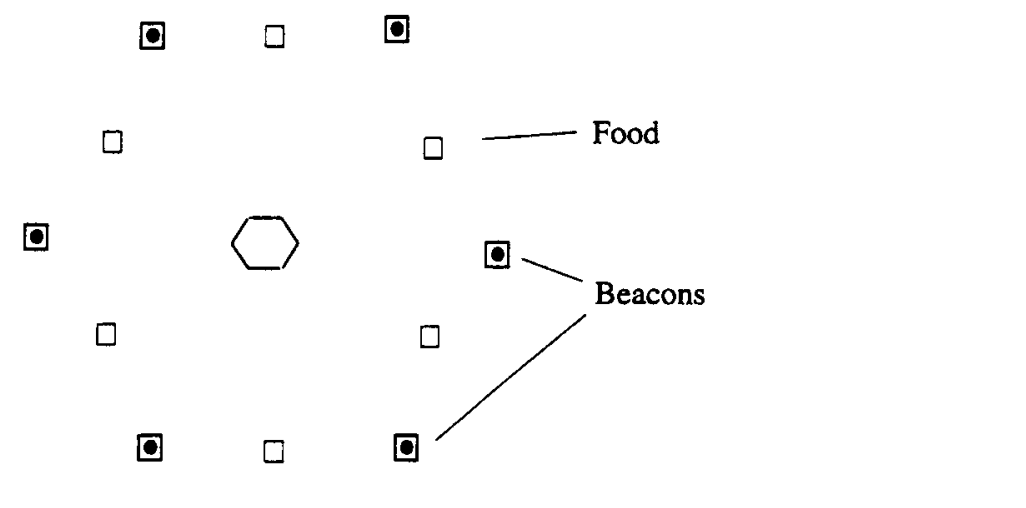

MISALIGNED BEACONS

Figure 7. The configuration of paths and beacons in experimental treatments in Experiment 2. 
tween cups, and no paths were present ( $n=10$ ); and (3) paths and beacons-six paths led from the center to the food cups, as in the paths-to-food treatment, and six $45.72-\mathrm{cm}$-tall beacons were directly next to the six food cups $(n=9)$. The planned comparisons between the paths-and-beacons treatment of Experiment 2 and the paths-tofood treatment of Experiment 1 were conducted with unpaired $t$ tests.

\section{Results}

General behavior. As in Experiment 1, the rats in Experiment 2 readily explored the maze and found food. In addition, the mean number of novel cups in the first six cup visits and the mean number of pellets per meter increased with time in all treatments. The number of novel cups in the first six cup visits showed significant correlations with the number of pellets per meter in 25 of 29 rats, which is a result of the lack of a difference in total distance traveled among treatments $[F(2,26)=.939, p=.404]$.

The influence of beacons on foraging efficiency. The mean number of pellets per meter among treatments differed in the first 4 days $[F(2,26)=6.936, p=.004$; see below], but the number of pellets per meter did not differ between the beacons-to-food and the misaligned-beacons treatments in the first 4 days $(p=.913)$. The mean number of pellets per meter did not differ among treatments in the final 4 days $[F(2,26)=2.640, p=.09$; Figure 8].

The frequency of approach to beacons. The rats in the misaligned-beacons treatment approached the beacons an average of $3.2( \pm 0.7)$ times per session in the first 4 days and $1.8( \pm 0.2)$ times per session in the final 4 days of the experiment. The number of beacon approaches did not differ significantly between the first 4 and the final 4 days [paired $t$ test, $t(9)=1.436, p=.185$ ], nor did the mean number of cup approaches per meter traveled in the open differ between the first $4(0.147 \pm 0.023)$ and the final 4 $(0.106 \pm 0.028)$ days [paired $t$ test, $t(9)=0.879, p=.402$ ]

The relative influence of paths and beacons on foraging efficiency. Initially, when paths and beacons both

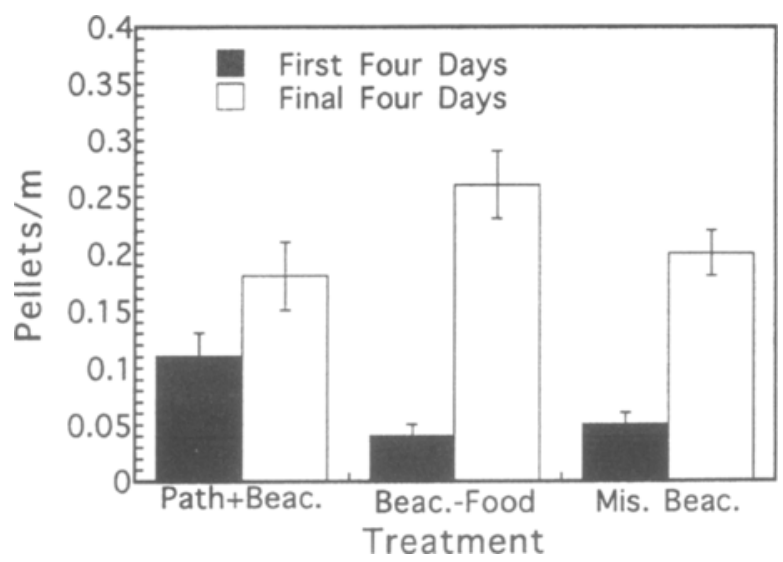

Figure 8. The mean number of pellets per meter in the first 4 and final 4 days of Experiment 2 (Path+Beac., paths and beacons; Beac.-Food, beacons to food; Mis. Beac., misaligned beacons).

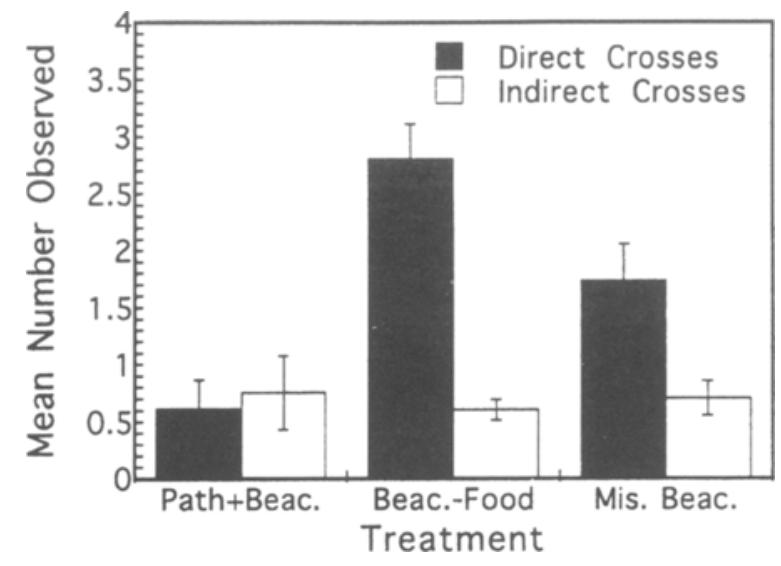

Figure 9. The mean number of direct and indirect crosses observed up to the sixth cup visit in the final 4 days of Experiment 2 (Path+Beac., paths and beacons; Beac.-Food, beacons to food; Mis. Beac., misaligned beacons).

led to food, rats displayed significantly higher mean number of pellets per meter than they did when only beacons led to food ( $p=.006$; Figure 8$)$. Therefore, paths facilitated a more economical acquisition of food in the first 4 days. By the final 4 days, however, the rate of intake was indistinguishable between the paths-and-beacons and beaconsto-food treatments. In the first 4 days, the mean number of pellets per meter was significantly higher in the paths-tofood treatment in Experiment 1 than in the beacons-tofood treatment [planned comparison, $t(16)=2.612, p=$ $.019]$. Therefore, paths initially facilitated a more efficient acquisition of food than did beacons. There was no significant difference in the rate of energy intake between these two treatments in the final 4 days [planned comparison, $t(16)=$ $1.690, p=.110]$. The mean number of pellets per meter did not differ between the paths-to-food and the paths-andbeacons treatments in either the first 4 [planned comparison, $t(15)=0.845, p=.411$ ] or the last 4 [planned comparison, $t(15)=0.432, p=.672$ ] days.

The number of direct crosses differed among treatments $[F(2,26)=11.964, p<.001]$. Just as the number of direct crosses was significantly higher in the short-paths treatment than the paths-to-food treatment, the number of direct crosses was significantly higher in the beacons-tofood $(p<.001)$ and misaligned-beacons $(p=.050)$ treatments than in the paths-and-beacons treatment (Figure 9). Therefore, in the absence of paths, the rats in the beaconsto-food and misaligned-beacons treatments achieved a rate of energy intake similar to that of the rats in the pathsand-beacons treatment by the final 4 days by following a cross-based foraging strategy.

Linear regressions between the mean number of direct crosses and the mean number of pellets per meter in the final 4 days in the individual treatments revealed that these variables showed significant positive correlations in treatments in which there were no paths but not in the treatment in which there were paths and beacons (Table 1). 


\section{Discussion}

The influence of beacons on foraging efficiency. The directness with which paths led to food influenced initial foraging efficiency (Experiment 1). Foraging efficiency was not influenced by how closely beacons were located to food, however. This provides evidence that paths had a more salient effect on foraging behavior than did beacons in the initial days of the experiment.

The frequency of approach to beacons. The mean number of approaches to beacons in the misaligned-beacons treatment in the first 4 days was 3.2. Because the rats could sometimes approach beacons even in random travel, this number does not support the hypothesis that rats have a strong predisposition to approach beacons with the physical characteristics of those used in this study.

The relative influence of paths and beacons on foraging effectiveness. When both paths and beacons led to food, the rats were able to achieve higher rates of energy intake than when only beacons led to food, which suggests that paths that lead to food do initially facilitate the efficient acquisition of food. That this difference in rate of energy intake disappeared by the final 4 days shows that, with experience, rats can learn to find food economically in the absence of paths. When both paths and beacons led to food, the rats did not achieve higher rates of energy intake than they did when only paths led to food. Therefore, beacons and paths do not provide a synergy of orientation cues that allows more efficient foraging than do paths alone.

When paths alone led to food, the rats displayed a higher initial rate of energy intake than when beacons alone led to food, which indicates that paths alone had more of an effect on efficiency than did beacons alone in the first 4 days. This difference disappeared by the final 4 days of the experiment, however, suggesting once again that, in the absence of paths as reliable predictors of food, rats can learn to orient to food by other means.

\section{GENERAL DISCUSSION}

The results of the present study demonstrate that travel along paths that lead to food can facilitate efficient foraging. Our results also establish that paths have a greater influence on foraging efficiency than do beacons and that, when paths and beacons both lead to food, rats do not enjoy a higher net rate of energy intake than when only paths lead to food. The present study also shows that how directly paths lead to food influences foraging effectiveness and that, as paths become less and less direct in their approach to food, they serve less and less as orientation cues to food. Our results indicate that, whereas rats may have a general tendency to follow trails, this predisposition can be overridden by energetic considerations. In addition, our results suggest that rats do not have a strong predisposition for approaching beacons with the physical characteristics of those in this study, possibly because they did not provide overhead cover such as that provided by trees and bushes.
In the last 4 days of both experiments, there were significant differences in the mean number of direct crosses, but there were not significant differences in the mean number of pellets per meter. We also observed differences in the correlation between the number of direct crosses and the mean number of pellets per meter. The observed differences in the number of direct crosses and the differences in correlations between the number of direct crosses and the number of pellets per meter show that the rats in different environmental conditions were using different strategies to achieve the same overall efficiency. For example, the rats in the short-paths, beacons-to-food, and misaligned-beacons treatments seemed to follow a crossbased foraging strategy, the rats in the paths-to-food and paths-and-beacons treatments seemed to follow a pathbased strategy, and the rats in the crooked-paths treatment seemed to follow an intermediate paths-and-indirectcrosses strategy.

The results of the present study suggest that rats do not follow paths exclusively as a way to reduce the risk of predation. If predator avoidance were the sole selective pressure favoring path following, we would not have observed differences in the proportion of path following between the paths-to-food and misaligned-paths treatments. However, our results certainly do not rule out predator avoidance as a selective benefit of path following. In fact, it is likely that energetic advantages and predator avoidance advantages have both contributed to the development of path following in Norway rats (see Botteron \& Arditi, 1991). The present study concentrated on economic and orientation issues. Tests employing empirical manipulations with predator models and comparative analyses of rodent species with different degrees of risk of predation in the wild would provide specific information on the extent to which predation risk has shaped path following behavior (see, e.g., Cassini, 1991; MacWhirter, 1991).

The results of the present study indicate that the orientation patterns and rates of energy intake of rats in an open arena are influenced by the presence of paths, the proximity of the paths to food cups, and the length of the paths. Furthermore, we observed that rats in different environmental conditions can adopt different strategies of orienting to food to achieve the same overall foraging efficiency. Further experiments are needed that provide rats with a wide range of potential behavioral choices, such as are found in open-arena studies. Such studies will offer advances in the understanding of the full range of foraging behaviors present in Norway rats, of how those behaviors are organized, and of the way in which those behaviors contribute to foraging efficiency and predator avoidance.

\section{REFERENCES}

Ash, M., \& Roberts, W A. (1992). Central-place foraging by rats on the radial maze The effects of patch size, food distribution, and travel time. Animal Learning \& Behavior, 20, 127-134.

BEL.L, W. J (1991). Searching behaviour. The behavioural ecology of finding resources London Chapman \& Hall. 
Botteron, B., \& Arditi, R. (1991). Central place foraging in nonpatchy habitats. Biometrical Journal, 33, 875-892.

CALhoun, J. B. (1962). The ecology and sociology of the Norway rat (Publication 1008). Bethesda, MD: U.S. Department of Health, Education, and Welfare.

CASSINI, M. H. (1991). Foraging under predation risk in the wild guinea pig Cavia aperea. Oikos, 62, 20-24.

FANSELOW, M. S., \& LESTER, L. S. (1988). A functional behavioristic approach to aversively motivated behavior: Predatory imminence as a determinant of the topography of defensive behavior. In R. C. Bolles \& M. D. Beecher (Eds.), Evolution and learning (pp. 185-210). Hillsdale, NJ: Erlbaum.

Hoffman, C. M., Timberlake, W., Gont, R., \& LefFel, J. (1997). Foraging in the laboratory: Do radial-arm mazes elicit natural foraging behavior? Unpublished manuscript.

MacArthur, R. H., \& Pianka, E. R. (1966). On optimal use of a patchy environment. American Naturalist, 100, 603-609.

MACWHIRTER, R. B. (1991). Effects of reproduction on activity and foraging behaviour of adult female Columbian ground squirrels. Canadian Journal of Zoology, 69, 2209-2216.

Olton, D. S., \& Samuelson, R. J. (1976). Remembrance of places passed: Spatial memory in rats. Journal of Experimental Psychology Animal Behavior Processes, 2, 97-116.
Roche, J. P. (1996). Patch-leaving decisions in black-capped chickadees. Animal Behaviour, 52, 289-298.

Roche, J. P., StubBs, D. A., \& Gl.ANZ, W. E. (1996). Assessment and choice: An operant simulation of foraging in patches. Journal of the Experimental Analysis of Behavior, 66, 327-347.

Schacter, G. B., Phelps, M. T., Brodbeck, D. R., Mogenson, G. J., \& ROBERTS, W. A. (1991). Disruption of central-place foraging in the rat following lesions of the dorsomedial thalamic nucleus. Psychobiology, 19, 91-99.

SCHOENER, T. W. (1971). Theory of feeding strategies. Annual Review of Ecology \& Systematics, 2, 369-404.

SoKal, R. R., \& RohlF, F. J. (1995). Biometry. New York: Freeman. STEPHENS, D. W. (1990). Foraging theory: Up, down, and sideways. Studies in Avian Biology, 13, 444-454.

StEPHENS, D. W., \& KReBS, J. R. (1986). Foraging theory. Princeton: Princeton University Press.

Telle, H. J. (1966). Beitrage zur Kenntnis der Vehaltensweise von Ratten, vegleichend dargestellt bei, Rattus norvegicus and Rattus rattus. Zeitschrift für angewandte Zoologie, 48, 97-129.

(Manuscript received January 2, 1997; revision accepted for publication July 25, 1997.) 